\title{
Chasing the Major Sphingolipids on Earth: Automated Annotation of Plant Glycosyl Inositol Phospho Ceramides by Glycolipidomics
}

\author{
Lisa Panzenboeck ${ }^{1}\left(\mathbb{D}\right.$, , Nina Troppmair ${ }^{1}$, Sara Schlachter ${ }^{1}$, Gunda Koellensperger ${ }^{1,2,3}$,

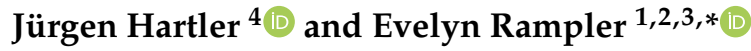 \\ 1 Department of Analytical Chemistry, Faculty of Chemistry, University of Vienna, Waehringer Str. 38, \\ 1090 Vienna, Austria; lisa.panzenboeck@univie.ac.at (L.P.); a01556591@unet.univie.ac.at (N.T.); \\ a11774156@unet.univie.ac.at (S.S.); gunda.koellensperger@univie.ac.at (G.K.) \\ 2 Vienna Metabolomics Center (VIME), University of Vienna, Althanstraße 14, 1090 Vienna, Austria \\ 3 Chemistry Meets Microbiology, University of Vienna, Althanstraße 14, 1090 Vienna, Austria \\ 4 Institute of Pharmaceutical Sciences, University of Graz, Universitätsplatz 1/I, 8010 Graz, Austria; \\ juergen.hartler@uni-graz.at \\ * Correspondence: evelyn.rampler@univie.ac.at; Tel.: +43-1-4277-52381
}

Received: 20 August 2020; Accepted: 16 September 2020; Published: 19 September 2020

\begin{abstract}
Glycosyl inositol phospho ceramides (GIPCs) are the major sphingolipids on earth, as they account for a considerable fraction of the total lipids in plants and fungi, which in turn represent a large portion of the biomass on earth. Despite their obvious importance, GIPC analysis remains challenging due to the lack of commercial standards and automated annotation software. In this work, we introduce a novel GIPC glycolipidomics workflow based on reversed-phase ultra-high pressure liquid chromatography coupled to high-resolution mass spectrometry. For the first time, automated GIPC assignment was performed using the open-source software Lipid Data Analyzer (LDA), based on platform-independent decision rules. Four different plant samples (salad, spinach, raspberry, and strawberry) were analyzed and the results revealed 64 GIPCs based on accurate mass, characteristic MS2 fragments and matching retention times. Relative quantification using lactosyl ceramide for internal standardization revealed GIPC $\mathrm{t} 18: 1 / \mathrm{h} 24: 0$ as the most abundant species in all plants. Depending on the plant sample, GIPCs contained mainly amine, $\mathrm{N}$-acetylamine or hydroxyl residues. Most GIPCs revealed a Hex-HexA-IPC core and contained a ceramide part with a trihydroxylated t18:0 or a t18:1 long chain base and hydroxylated fatty acid chains ranging from 16 to 26 carbon atoms in length (h16:0-h26:0). Interestingly, four GIPCs containing t18:2 were observed in the raspberry sample, which was not reported so far. The presented workflow supports the characterization of different plant samples by automatic GIPC assignment, potentially leading to the identification of new GIPCs. For the first time, automated high-throughput profiling of these complex glycolipids is possible by liquid chromatography-high-resolution tandem mass spectrometry and subsequent automated glycolipid annotation based on decision rules.
\end{abstract}

Keywords: glycolipidomics; GIPC; glycosyl inositol phospho ceramides; Lipid Data Analyzer; lipidomics; sphingolipids; ultra-high pressure liquid chromatography; high-resolution mass spectrometry; LC-MS; automated annotation

\section{Introduction}

The sphingolipidome of plants contains glycosyl inositol phospho ceramides (GIPCs), glycosylceramides and ceramides, whereas sphingomyelin, globosides, sulfatides or gangliosides are absent. GIPCs were characterized as the major sphingolipid on earth due to their high abundance 
in plants and fungi, which comprise a large portion of the biomass of the biosphere [1]. GIPCs were first described more than 60 years ago as "phytoglycolipids" [2]. The total plant lipid content can consist of up to $40 \%$ GIPCs [3]. The structure of these plant sphingolipids has three major subunits: (1) a polar inositol containing part, (2) the sphingoid backbone with a long-chain base (amino-alcohol) linked by an amide bond to a (3) fatty acyl chain moiety [2,4]. The terms $d, t$ and $q$ refer to the hydroxylation state of the whole ceramide or long-chain base (LCB) moiety, ranging from two (d) to four $(q)$ hydroxy groups. The term $h$ denotes a hydroxylation of the fatty acyl group (i.e., the ceramide moiety q40:1 can correspond to a t18:1 LCB connected to a h22:0 fatty acyl). Di- and trihydroxylation of LCBs with $\mathrm{t} 18: 0, \mathrm{t} 18: 1$ (8Z and $8 \mathrm{E}$ ) (the main sphingoid base in some species), and d18:0, d18:1(8Z and $8 \mathrm{E}), \mathrm{d} 18: 2$ (4E/8Z and $4 \mathrm{E} / 8 \mathrm{E})$ and fatty acid components varying in chain-length, saturation and hydroxylation state (h16:0-h26:1, 20:0 to 28:0) have been reported in plant GIPCs [5,6]. Different GIPC core structures were determined from higher plants ranging from simple high-abundant A-series species with Hex-HexA-IPC and HexN(Ac)-HexA-IPC (Hex = hexose, HexA = hexuronic acid, IPC = inositol phospho ceramide, HexN = hexosamine, and HexNAc $=\mathrm{N}$-acetyl hexosamine) to low abundant F-series species containing several arabinoses and hexoses [3,7]. Despite the fact that GIPCs are an integral part of the plant plasma membrane, there is still little knowledge concerning its molecular organization and the way this organization is involved in signaling processes necessary for cellular adaptation [1]. To understand the interplay of GIPCs with different enzymes and their detailed function in the plasma membrane in plants, comprehensive structural information provided by observation tools such as NMR or MS are necessary.

Even though GIPCs were discovered 60 years ago, their analysis remains challenging due to the lack of available standards, automated annotation software and reference databases. For example, CHEBI [8] does not provide any GIPCs and the comprehensive LIPID MAPS Structure Database (LMSD) contains only one GIPC (A-NH $-\mathrm{t} 18: 1 / \mathrm{h} 24: 0)$ [9]. As GIPCs consist of a sugar head group linked to a lipid subunit causing amphiphilic properties, they are neither well covered by common glycomics nor lipidomics workflows. Consequently, specialized glycolipidomics analysis strategies are required, e.g., applying a mixture of 2-propanol (IPA), hexane and water [10]. The combination of liquid chromatography and mass spectrometry (LC-MS) has been used due to its unprecedented potential to annotate GIPCs by $m / z$, retention time and fragmentation pattern [7,11]. Unambiguous GIPC identification requires both retention time evaluation and detection of structural subunits by tandem mass spectrometry (MS2), due to the absence of commercial standards. Most GIPC LC-MS-based analysis workflows were performed almost a decade ago by electrospray ionization followed by analysis with low resolution mass spectrometers (QQQ, QTRAP) [7,11]. Meanwhile, high-resolution mass spectrometers (such as TOF, orbitrap, FTICR) have been established with up to 1,000,000 resolution enabling GIPC analysis by accurate mass [12]. Additionally, ultra-high pressure liquid chromatography (up to 1500 bar) with sub 2- $\mu$ m particles provides high chromatographic resolution and excellent sensitivity. Up to now, GIPC analysis has been performed by tedious manual annotation and curation $[1,7,12,13]$ and expert knowledge was necessary to interpret glycosphingolipid tandem mass spectrometry fragmentation patterns [14-16]. The instrumentation advancements of the recent years paved the way for automated high-throughput GIPC analysis. In this work, a variety of plants, i.e., iceberg lettuce (Lactuca sativa var. capitata nidus tenerimma), deep frozen spinach (Spinacia oleracea), raspberries (Rubus idaeus), and strawberries (Fragaria) were analyzed by the combination of reversed-phase (RP) ultra-high pressure liquid chromatography (UHPLC) and high-resolution mass spectrometry (HRMS). For the first time, automated GIPC annotation will be performed using the Lipid Data Analyzer (LDA) and platform-independent decision rules [17].

\section{Results}

Here we describe a novel workflow by RP-HRMS/MS using the open-source program LDA [17] for automated GIPC assignment. Method development considerations and guidelines for the automated structural analysis of GIPCs are provided. Finally, we test the developed glycolipidomics workflow 
for different plant samples, leading to a reference database of GIPCs, including fragmentation and retention time information.

\subsection{Method Development for Automated GIPC Assignment}

GIPCs were extracted by a mixture of IPA, n-hexane and water [18]. So far, most LC-MS-based GIPC chromatographic separations relied on the use of tetrahydrofuran (THF) containing solvents [7,11-13]. However, the usage of THF in the eluent system has some drawbacks: (1) it is aprotic and cannot donate a proton; thus, for ionization, pairing with a protic solvent (usually water) is necessary; (2) it can attack tubing (especially PEEK tubing); (3) it tends to polymerize (usually in APCI mode); and (4) it is highly flammable. In order to avoid the use of THF, we developed a novel GIPC method based on RP-HRMS/MS, facilitating a $30 \mathrm{~min}$ isopropanol gradient (detailed information can be found in the Materials and Methods Section 4.3). GIPC detection was performed using both negative and positive electrospray ionization and high-resolution Orbitrap MS (see Materials and Methods Section 4.4). Importantly, GIPC analysis requires relatively high RF voltages (S-lens RF level of 45) to ensure efficient transport of medium size glycolipids in the mass spectrometer. Figure 1 shows the extracted ion chromatogram of GIPCs in salad samples analyzed by RP-HRMS, based on data-dependent MS2 (ddMS2) in positive and negative ion modes. The GIPCs displayed in Figure 1 belong to the A-series (Hex(R1)-HexA-IPC) with R1 being a hydroxyl group and the ceramide portion consisting of a hydroxylated saturated fatty acyl chain attached to a t18:1 long chain base.

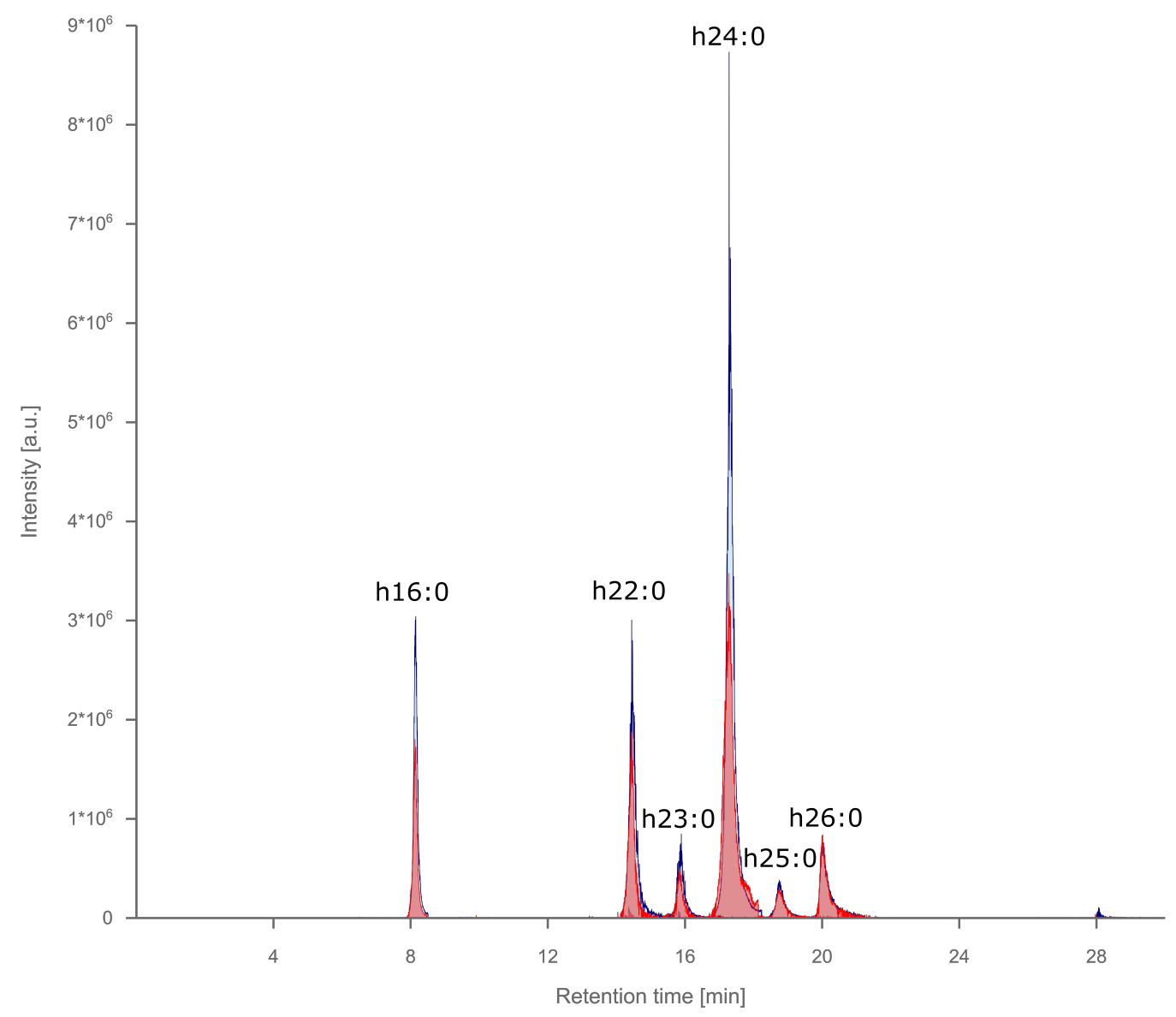

Figure 1. Extracted ion chromatogram of glycosyl inositol phospho ceramides (GIPCs) in salad samples analyzed by RP-HRMS/MS analysis using ddMS2 in positive (red) and negative (blue) ion modes. Assigned GIPCs belong to the Hex-HexA-IPC series with a t18:1 long-chain base (LCB) and varying chain length of the hydroxylated saturated fatty acids. Retention times coincided in positive and negative ion modes. Increasing carbon numbers result in belated elution. 
As no commercial standards are available, GIPC assignment has to be conducted with caution. In such a situation, the use of the equivalent carbon number model (ECN) is required [19,20]. The ECN model originates from state of the art lipidomics workflows and is based on elution orders observed in RP columns: (1) longer fatty acid chains will increase the retention time (see Figure 1) and (2) more double bonds will decrease the retention time [21] (see Table S1). To increase the level of confidence in GIPC annotation, we accepted only GIPCs that: (1) were detectable by accurate mass ( \pm 5 ppm) in MS1 at the same retention time in both positive and negative ion modes (Figure 1); (2) showed MS2 spectra with characteristic fragments for the ceramide and sugar part in at least one ion mode and; (3) fulfilled the ECN model.

\subsection{Structural Elucidation and GIPC Annotation Based on MS2 Information}

In this work, we introduce the first automated GIPC annotation workflow based on structural information provided by acquired MS2 spectra. Structural analysis and automated GIPC annotation was performed based on a set of in-house developed decision rules for the freely available software LDA [17,22]. As no standards were available, blank extractions (no GIPC annotations found) and GIPC annotations in salad [13] and spinach [12] reported in the literature were used to validate GIPC assignments (Figure 1, Table A1 and Table S2). Various LCBs (d18:0, d18:1, d18:2, t18:0, and t18:1) and fatty acids (FAs) (16-26) with or without hydroxylation have been reported [5,13]. Moreover, R1 in Figure 2A can either be a hydroxyl $(\mathrm{OH})$, an amine $\left(\mathrm{NH}_{2}\right)$ or an $\mathrm{N}$-acetylamine (NAc) group, increasing the number of putative GIPCs even within a single series.

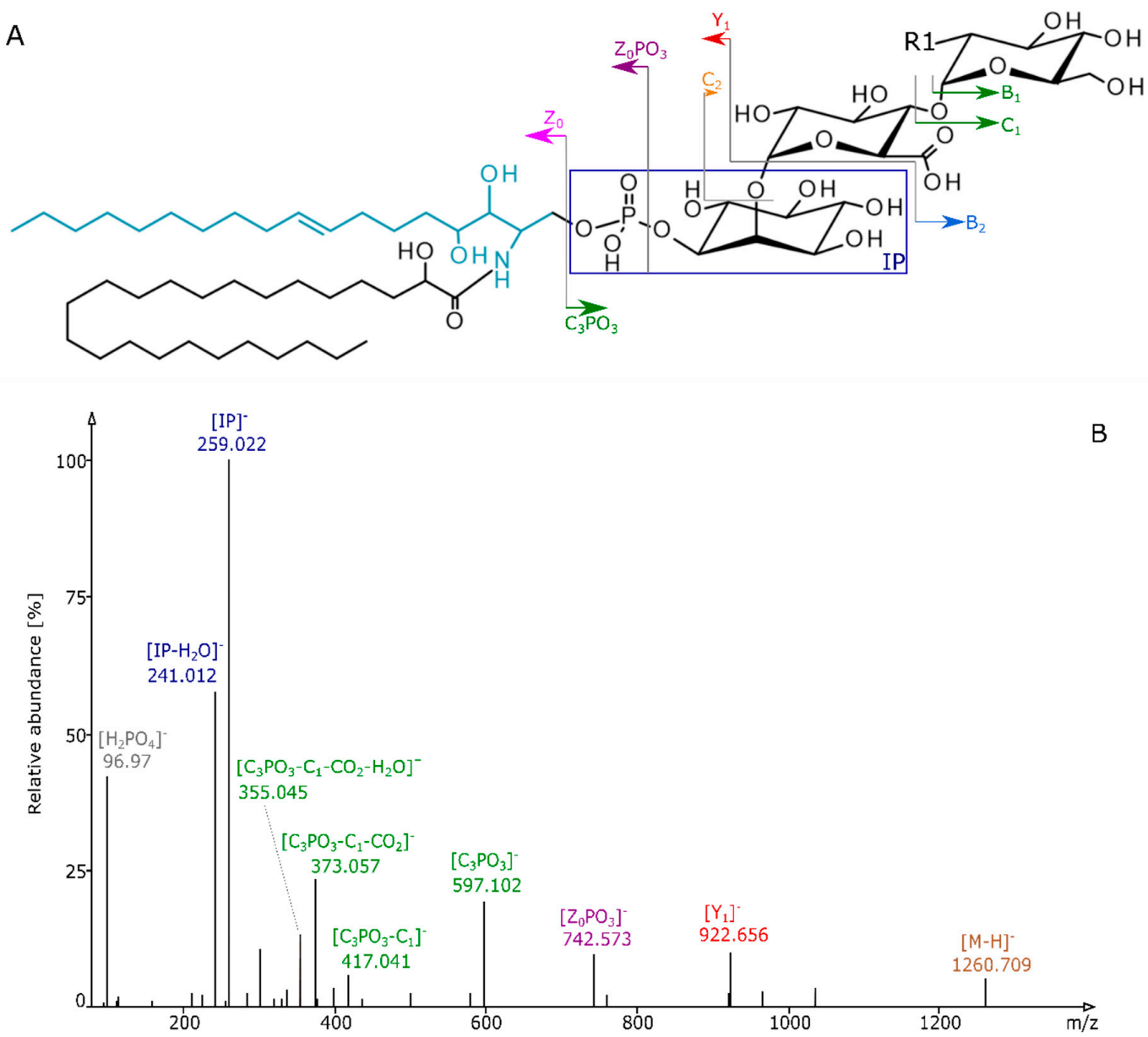

Figure 2. Cont. 


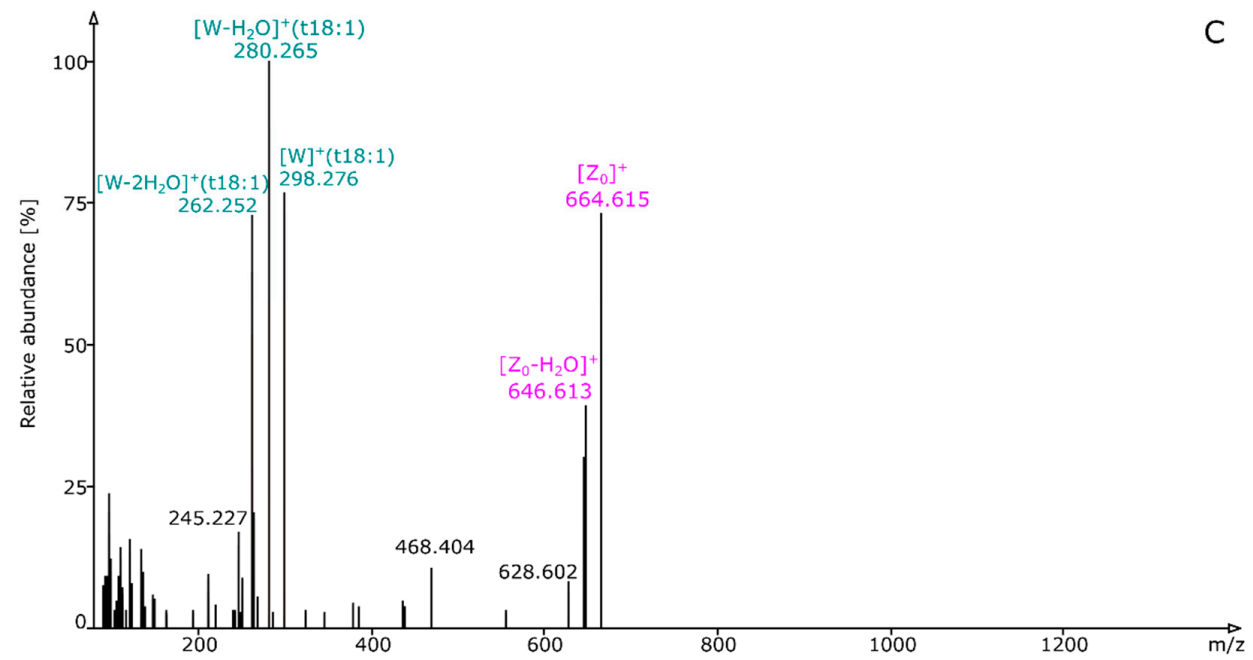

Figure 2. Overview of the GIPC fragmentation for the example of GIPC A-OH-t18:1/h24:0 in salad: (A) The fragment assignment of GIPC A-OH-t18:1/h24:0 (adapted from [23]). The W fragment is shown in a light blue color. Please note that a full structural characterization is not possible by RP-HRMS/MS, (B) The product ion spectrum in negative ion mode at $\mathrm{m} / \mathrm{z} 1260.7237$, showing characteristic fragments $\mathrm{m} / \mathrm{z}$ 241 and $259,355,373$ and 417 . The sugar head group was confirmed by the $\left[\mathrm{C}_{3} \mathrm{PO}_{3}\right]^{-}$fragment $(\mathrm{m} / \mathrm{z}$ $597, \mathrm{R} 1=\mathrm{OH}) \cdot\left[\mathrm{Z}_{0} \mathrm{PO}_{3}\right]^{-}$and $\left[\mathrm{Y}_{1}-\mathrm{H}\right]^{-}$fragments prove the ceramide moiety. $(\mathrm{C})$ The positive ion mode ddMS2 spectrum of the $[\mathrm{M}+\mathrm{H}]^{+}$precursor, exhibiting the $[\mathrm{W}]^{+},\left[\mathrm{W}-\mathrm{H}_{2} \mathrm{O}\right]^{+}$and $\left[\mathrm{W}-2 \mathrm{H}_{2} \mathrm{O}\right]^{+}$fragments at $m / z 298,280$ and 262, which are characteristic for the t18:1 LCB.

The final decision rule set was based on well-defined fragments (fragment rules) and their intensity relationships (intensity rules) (Folder S1). The characteristic fragments $[\mathrm{IP}]^{-}(\mathrm{m} / \mathrm{z} 259)$ and $\left[\mathrm{IP}-\mathrm{H}_{2} \mathrm{O}\right]^{-}$ $(\mathrm{m} / \mathrm{z} 241)$ are mandatory in negative ion mode (e.g.: Figure 2B). However, these fragments are not specific, since they are produced by other phosphoinositol-containing lipids too. Thus, for a confident identification, negative or positive ion mode fragments indicating the sugar or ceramide part have to be detected.

In the majority of cases (see level 2 annotations, Table A1 and Table S2), MS2 spectra with GIPC fragmentation patterns were detected in both negative and positive mode. Depending on the fragmentation pattern and the level of confidence [24] of the structural elucidation, GIPCs are assigned as either: (1) series-R1-hydroxylation stage-carbon number (LCB + FA)-number of double bonds (LCB + FA) if the exact ceramide composition is not known or (2) series-R1-LCB/FA. Figure 2B displays an exemplary ddMS2 spectrum of A-OH-q42:1 with $\mathrm{m} / \mathrm{z} 1260.7237$, in salad recorded in negative ion mode. The positive ion mode fragmentation pattern of the $[\mathrm{M}+\mathrm{H}]^{+}$precursor $(\mathrm{m} / \mathrm{z} 1262.7389$, Figure $2 \mathrm{C})$ revealed further structural details, based on the identification of $[\mathrm{W}]^{+},\left[\mathrm{W}-\mathrm{H}_{2} \mathrm{O}\right]^{+}$and $\left[\mathrm{W}-2 \mathrm{H}_{2} \mathrm{O}\right]^{+}$ fragments, indicating an A-OH-t18:1/h24:0 GIPC. Additional GIPC confirmation is possible by $\mathrm{Z}_{0}$ fragments $\left(\left[\mathrm{Z}_{0}\right]^{+},\left[\mathrm{Z}_{0}-\mathrm{H}_{2} \mathrm{O}\right]^{+}\right)$of the $[\mathrm{M}+\mathrm{H}]^{+}$precursor and by the sodium adduct $[\mathrm{M}+\mathrm{Na}]^{+}$(Figure $\left.\mathrm{A} 1\right)$, where sugar fragments are readily observable. GIPCs were annotated based on single ionization information only if (1) in negative ion mode in addition to the apparent $[\mathrm{IP}]^{-} /\left[\mathrm{IP}-\mathrm{H}_{2} \mathrm{O}\right]^{-} /\left[\mathrm{H}_{2} \mathrm{PO}_{4}\right]^{-}$ fragments at $m / z 259,241$ and 97, other characteristic fragments were detectable e.g., $\left[\mathrm{C}_{3} \mathrm{PO}_{3}\right]^{-}$ $\left(m / z 596-\mathrm{R} 1=\mathrm{NH}_{2}, m / z 597-\mathrm{R} 1=\mathrm{OH}, m / z 638-\mathrm{R} 1=\mathrm{NAc}\right),\left[\mathrm{C}_{3} \mathrm{PO}_{3}-\mathrm{C}_{1}-\mathrm{CO}_{2}\right]^{-}(\mathrm{m} / \mathrm{z} 373)$ or $\left[\mathrm{C}_{3} \mathrm{PO}_{3}-\mathrm{C}_{1}-\mathrm{CO}_{2}-\mathrm{H}_{2} \mathrm{O}\right]^{-}(\mathrm{m} / \mathrm{z} 355)$ or (2) in positive ion mode the $[\mathrm{IP}]^{+}(\mathrm{m} / \mathrm{z} 261) /[\mathrm{IP}+\mathrm{Na}]^{+}(\mathrm{m} / z 283)$ and fragments indicating the ceramide moiety (e.g., $\mathrm{Z}_{0}$ ) were identified by LDA. The detailed fragment information used for GIPC annotation can be found in Table S2.

GIPC annotation can be hampered by the presence of isobaric masses for $\mathrm{qX}: \mathrm{Y} \mathrm{NH}_{2}$ and $\mathrm{t}(\mathrm{X}-$ 2):(Y - 1) NAc (where $X$ refers to the carbon number (LCB + FA) and Y refers to the number of double bonds (LCB + FA), respectively). This may result in false positive GIPC identifications, because these 
classes share the same characteristic fragments $m / z 241,259,355,373$ and 417 . The correct structural elucidation is possible if additional fragments such as $\left[\mathrm{C}_{3} \mathrm{PO}_{3}\right]^{-}\left(\mathrm{R} 1=\mathrm{OH}-\mathrm{m} / z 597, \mathrm{R} 1=\mathrm{NH}_{2}\right.$ $m / z 596, \mathrm{R} 1=\mathrm{NAc}-m / z 638$ ) in negative ion mode or if LCBs in positive ion mode can be identified based on $[\mathrm{W}]^{+},\left[\mathrm{W}-\mathrm{H}_{2} \mathrm{O}\right]^{+}$and $\left[\mathrm{W}-2 \mathrm{H}_{2} \mathrm{O}\right]^{+}$fragments. In the ddMS2 spectra of the $[\mathrm{M}+\mathrm{H}]^{+}$-precursor, trihydroxylated LCBs are characterized by the presence of three $\mathrm{W}$ fragments $\left([\mathrm{W}]^{+},\left[\mathrm{W}-\mathrm{H}_{2} \mathrm{O}\right]^{+}\right.$and $\left.\left[\mathrm{W}-2 \mathrm{H}_{2} \mathrm{O}\right]^{+}\right)$, such as t18:0 ( $\mathrm{m} / \mathrm{z} 300,282$ and 264) and $\mathrm{t} 18: 1(\mathrm{~m} / \mathrm{z} 298,280,262)$, while dihydroxylated species miss the $\left[\mathrm{W}-2 \mathrm{H}_{2} \mathrm{O}\right]^{+}$fragment, e.g., d18:0 $(\mathrm{m} / \mathrm{z} 284,266), \mathrm{d} 18: 1(\mathrm{~m} / \mathrm{z} 282,264)$ and d18:2 $(\mathrm{m} / \mathrm{z} 280$, 262). As such, both LCB hydroxylation levels can be clearly distinguished.

\subsection{Analysis of Different Plant GIPCs by UHPLC-HRMS Suggesting t18:2 LCB}

The novel RP-HRMS/MS and GIPC annotation workflow was used to analyze different plant samples, namely salad (Lactuca sativa var. capitata nidus tenerimma), deep frozen spinach (Spinacia oleracea), raspberries (Rubus idaeus) and strawberries (Fragaria). As glycosphingolipid analysis is not negatively impacted by alkaline hydrolysis [10], alkaline hydrolysis was performed to simplify lipid profiles by removing the phospholipid background in the unknown plant samples (strawberry and raspberry, detailed information can be found in the Materials and Methods Section 4.2.2). Figure A2 shows the RP-HRMS/MS GIPC profile for the five most abundant GIPCs determined in spinach, strawberry and raspberry samples. For the sake of clarity, the five most abundant GIPCs in salad (A-NAc-t18:1/h24:0, A-NH - -t18:1/h24:0, A-OH-t18:1 h22:0 and h24:0, A-OH-t18:0/h24:0) are not displayed in Figure A2. Irrespective of the plant sample, the species group A-R1-t18:1/h24:0 was always the most abundant one. While in spinach R1 was always N-acetylamine (A-NAc-t18:1 h22:0 to h26:0) for the five dominating GIPCs, in strawberries the major GIPCs contained a hydroxyl group as R1 (A-OH-t18:1 h23:0 to h26:0 and A-OH-t18:0/h24:0). In contrast to that, raspberries had an amine group as $\mathrm{R} 1$ for four out of five shown GIPCs (A-NH $2-\mathrm{t} 18: 0 / \mathrm{h} 24: 0, \mathrm{~A}-\mathrm{NH}_{2}-\mathrm{t} 18: 1 \mathrm{~h} 22: 0$ and $\mathrm{h} 24: 0, \mathrm{~A}-\mathrm{NH}_{2}-\mathrm{t} 18: 2 / \mathrm{h} 24: 0$ and A-OH-t18:1/h24:0), emphasizing the structural diversity of GIPCs in different plants.

By analyzing different GIPCs, the NAc, $\mathrm{NH}_{2}$ and $\mathrm{OH}$-species from the A series could be detected (Figure 3A-C) with high confidence by (1) accurate determination of mass, (2) matching retention times of ion modes, (3) characteristic fragments and (4) the ECN model. We recommend checking isotopic patterns to avoid false positive hits. For a comprehensive overview of the annotated GIPCs see Table A1.

Due to the absence of commercially available GIPC standards, relative quantification of the individual species was performed using $\mathrm{C} 16$ lactosyl(B) ceramide (d18:1/16:0) as the internal standard. This compound is similar in structure (sugar and ceramide moiety) and retention time (14 min). Even though lactosyl ceramide (d18:1/16:0) may be present in plants, we could not detect it in our samples, thus, making it suitable as the internal standard in our workflow. Normalization by the internal standard (area ratio) and dry weight was performed for MS1-based relative quantification by Skyline [25] (Figure 3A-C). Estimated concentrations in the nmol to $\mu \mathrm{mol}$ range per gram dry weight were observed, which is consistent with the literature [12,18].

In summary, 64 GIPCs in salad (19), spinach (8), strawberry (10) and raspberry (27) were annotated (Table A1). Ranking of the GIPC annotations was performed according to the guidelines of the metabolomics society [24,26], leading to 48 level 2 (matching accurate masses and MS2 in negative and positive mode) GIPCs, 13 level 3 (MS2 in one ion mode with matching accurate masses in both ion modes) GIPCs and 3 level 3** (matching accurate masses in both ion modes, MS2 in one ion mode but lacking information on IP fragments in positive ion mode or lacking sugar information in negative ion mode) GIPCs. The annotations found in spinach and salad are in accordance with literature [12,13]. To the best of our knowledge, this is the first report on GIPCs in strawberries and raspberries. 

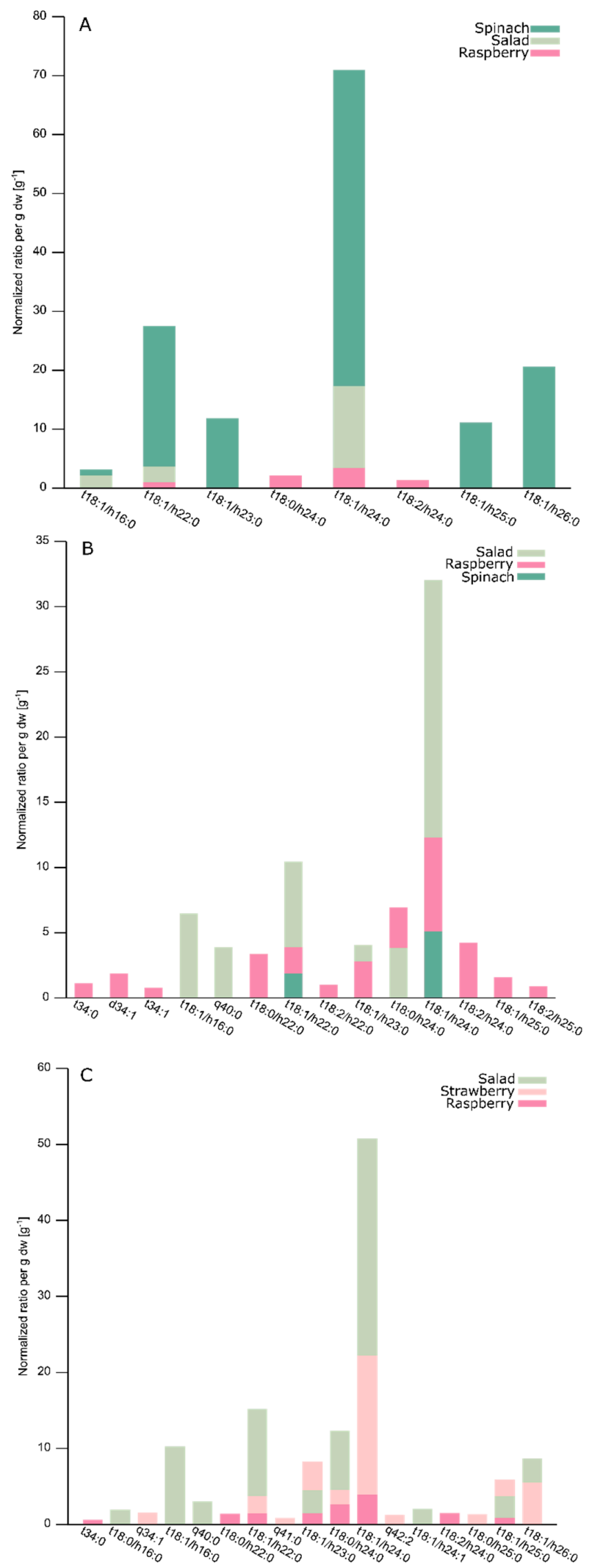

Figure 3. The normalized ratio per gram dry weight for annotated GIPCs in salad (light-green), spinach (green), strawberries (rose) and raspberries (dark-red), by using different substituents for the functional group R1: (A) NAc, (B) $\mathrm{NH}_{2}$, and (C) $\mathrm{OH}$ (more detailed information can be found in Table A1). 
Within the annotated GIPCs with structural information on LCB and fatty acyl composition, t18:1 followed by $\mathrm{t} 18: 0$ and $\mathrm{t}$ 18:2 were the most prominent LCBs in the analyzed plants (Table A1). For GIPCs containing $\mathrm{N}$-acetylamine residues $\mathrm{t} 18: 1$ was the most abundant LCB with regard to normalized ratios per gram dry weight (Figure 3A). The same holds true for the amine or hydroxyl group containing GIPCs, with additional high abundance of t18:0 LCBs (Figure 3B,C). While in spinach solely t18:1 LCBs were detected, salad, strawberries and raspberries show more variation in terms of LCB composition with presence of both $\mathrm{t} 18: 1$ and $\mathrm{t} 18: 0$ (Table A1).

Interestingly, besides the expected t18:0 and t18:1 LCBs ( $\left.\mathrm{R} 1=\mathrm{NAc}, \mathrm{NH}_{2}, \mathrm{OH}\right)$, we additionally annotated four t18:2 ( $\mathrm{R} 1=\mathrm{NAc}, \mathrm{NH}_{2}, \mathrm{OH}$ ) species in raspberries (Figure 3A-C). These annotations are verified by coinciding retention times in positive ion mode (Figure A3A), detection of characteristic fragments in MS2 spectra (Figure A3B,C) and conformity with the ECN model (Table S1). However, we could not find any report in the literature of $t 18: 2$ species, which can be explained as up to now no automated GIPC annotation was possible and t18:2 GIPC species were only detected in raspberries. As no standards are available, it is difficult to prove the presence of this species and further investigation is needed. A confirmed t18:2 LCB would indicate a much higher diversity in sphingolipids than anticipated in the past. Another hint for the complex nature of GIPCs in raspberries is the additional annotation of GIPCs with di- and trihydroxylated variants compared to all other analyzed plants.

Concerning LCB and fatty acyl combinations, $\mathrm{t} 18: 1 / \mathrm{h} 22: 0$ and $\mathrm{t} 18: 1 / \mathrm{h} 24: 0$ showed equal annotation numbers for $\mathrm{N}$-acetylamine or amine containing GIPCs (Figure 3A,B). Independent of the $\mathrm{NH}_{2}, \mathrm{OH}$ or NAc functional group, only two odd chain fatty acids (h23:0, h25:0) were detected and no fatty acids with a length from 17 to 21 carbon atoms were found. For the hydroxyl group containing GIPC variants, the combinations $\mathrm{t} 18: 1 / \mathrm{h} 22: 0, \mathrm{t} 18: 1 / \mathrm{h} 23: 0, \mathrm{t} 18: 0 / \mathrm{h} 24: 0, \mathrm{t} 18: 1 / \mathrm{h} 24: 0$ and $\mathrm{t} 18: 1 / \mathrm{h} 25: 0$ were found in equal annotation numbers. Overall, plant GIPCs with a combination of t18:1 LCB and a h24:0 fatty acyl moiety were the most abundant ones in terms of normalized ratios per gram dry weight (Figure 3A-C, Table A1).

\section{Discussion}

GIPCs are the major sphingolipids on earth [1]. Hence, it is important to understand their function and distribution in plants and fungi. However, GIPC analysis remains extremely challenging, as tailored extraction strategies for this glycolipid class are necessary. GIPC analysis is in its infancy due to the lack of standards and databases. In this work, we present the first automated high-throughput GIPC annotation workflow which is based on RP-HRMS/MS. By using a novel 30 min gradient based on isopropanol with a reversed-phase column, packed with sub 2- $\mu \mathrm{m}$ particles, fast GIPC analysis was possible at the same time avoiding standard eluent use of tetrahydrofuran. Four different plant samples were analyzed. For salad and spinach, literature information has been available [12,13], while for raspberry and strawberry, GIPC profiles were completely uncharacterized. Using strict filtering by (1) accurate mass determination $( \pm 5 \mathrm{ppm})$ with matching retention times for both ion modes in MS1, (2) MS2 spectra with characteristic fragments and (3) expected retention time series, we produced a database of 64 GIPCs (Table A1). As no GIPC standards are available, only GIPC annotation hits with level 2 and 3 confidence [24] were possible. The most prominent MS2 fragments for GIPCs are [IP] fragments in both ion modes $\left([\mathrm{H}]^{-}: m / z 241,259 ;[\mathrm{H}]^{+}: m / z 261 ;[\mathrm{Na}]^{+}: m / z 283\right)$. However, additional sugar or ceramide fragments are essential for correct GIPC annotation. The high MS2 mass range coverage $(\mathrm{m} / \mathrm{z} 65$ to 2500$)$ provided by the Orbitrap was beneficial to determine GIPC low mass fragments such as $m / z 79\left[\mathrm{PO}_{3}\right]^{-}$or $97\left[\mathrm{H}_{2} \mathrm{PO}_{4}\right]^{-}$, besides high mass precursors such as $1261[\mathrm{M}-\mathrm{H}]^{-}$ (Figure 2).

Relative quantification with the internal standard lactosyl ceramide revealed GIPC $\mathrm{t} 18: 1 / \mathrm{h} 24: 0$ as the most abundant species, independent of the plant sample. Depending on the plant sample, GIPCs contained mainly amine, N-acetyl or hydroxyl residues. Most GIPCs showed a Hex-HexA-IPC core with a trihydroxylated t18:0 or t18:1 long-chain base ceramide part a and hydroxylated fatty acid chains ranging from h16:0 to h26:0. Interestingly, in raspberry, four GIPCs contained $\mathrm{t} 18: 2$, which was not 
reported so far. This finding would suggest the existence of more complex sphingolipid species in nature than previously anticipated. Further analysis by orthogonal methods such as NMR, GC-MS or IMS and available GIPC standards would be necessary to confirm the presence of the t18:2 GIPC group. Different analytical strategies could also resolve potential isomeric species and provide comprehensive details on the sugar moiety present in GIPCs. Nevertheless, this example shows the power of this workflow to detect promising novel GIPC candidates in an automated fashion. In order to support LC-MS-based GIPC analysis in general, we provide the mass lists for GIPCs in positive and negative ion modes (Tables S3 and S4), as well as the fragmentation rules (Folder S1) for setting up the automated GIPC analysis by Lipid Data Analyzer. Even though we confirmed the GIPCs exclusively from the A-series, the presented strategy is also suitable to determine less or more complex GIPC series, such as 0, B, C, D, E and F. However, extended analytical workflows (e.g., multi-stage fragmentation/MSn) and additional software method development might be necessary. Precursor mass lists for positive ([M+ $\mathrm{H}]^{+}$) and negative $\left([\mathrm{M}-\mathrm{H}]^{-}\right)$ion modes comprising series 0-F, LCBs d18:0, d18:1, d18:2, t18:0 and t18:1 and fatty acyls h15:0-h26:0, h15:1-h26:1 and n20:0-n28:0 ( $\mathrm{n}=$ non-hydroxylated), as reported in the literature [5,13], can be found in Tables S5 and S6. In general, we believe that LC-HRMS/MSn combined with automated annotation based on decision rules will pave the way for more complex glycolipidomics profiling.

\section{Materials and Methods}

\subsection{Material}

The plant material used was derived from salad (Lactuca sativa var. capitata nidus tenerimma), deep frozen spinach (Spinacia oleracea), raspberries (Rubus idaeus) and strawberries (Fragaria). (A more detailed description of plant samples can be found in Table A2.)

All chemicals were of LC-MS grade. Acetonitrile (ACN), methanol (MeOH), IPA and water were bought from Honeywell (Offenbach, Germany) and n-hexane was bought from VWR (Vienna, Austria). Butylated hydroxytoluene (BHT) was purchased from Sigma-Aldrich (Vienna, Austria), ammonium formate (AF) from Sigma-Aldrich (Vienna, Austria) and formic acid from VWR (Vienna, Austria). C16 Lactosyl(ß) Ceramide (d18:1/16:0) (D-lactosyl-ß-1,1' N-palmitoyl-D-erythro-sphingosine) was purchased from Avanti Polar Lipids, Inc. (Alabaster, Albama, USA), was used as internal standard (IS) and dissolved in an appropriate amount of IPA to achieve a concentration of $100 \mu \mathrm{M}$.

\subsection{Sample Preparation}

Salad was manually cut into small pieces before being weighed into falcon tubes $(50 \mathrm{~mL}$, VWR, Vienna, Austria) using a CPA225D balance (Sartorius, Vienna, Austria). Raspberries and strawberries (whole fruits) were homogenized with a hand blender (Tefal/SEB, Ecully, France). Raspberries, strawberries and deep-frozen homogenized spinach were directly weighed into $10 \mathrm{~mL}$ glass vials (more details can be found in Table A2). In order to prevent potential oxidation of lipids, $3 \mathrm{~mL}$ of an approximately $0.01 \%$ BHT solution in IPA were added and samples were mixed. Subsequently $30 \mu \mathrm{L}$ IS were spiked into all samples except for one replicate (to test for potential IS presence in plants). Salad samples were homogenized using an ultra-turax (miccra d-1, Heitersheim, Germany) which was cleaned with $70 \%$ IPA and dried between the samples. In order to inhibit lipase activity, all samples were incubated at $75^{\circ} \mathrm{C}$ for 30 min under constant shaking [27]. The warm salad samples were subsequently transferred into glass vials. The following sections provide a detailed overview of the extraction strategies that were applied.

\subsubsection{One-Phase Extraction}

The extraction of GIPCs from salad and spinach was performed as previously reported [18] using a mixture of IPA, n-hexane and water. Amounts of $3.47 \mathrm{~mL}$ IPA, $0.6 \mathrm{~mL}$-hexane and $1.93 \mathrm{~mL}$ water were added to the salad and spinach samples. In order to ensure sufficient accessibility of the plant 
material, samples were vortexed and manually shaken prior to incubation at $60{ }^{\circ} \mathrm{C}$ for 15 min under constant shaking.

\subsubsection{One-Phase Extraction Combined with Alkaline Hydrolysis}

To avoid the occurrence of glycerophospholipids, which might reduce GIPC ionization efficiency and lead to potential false identifications, alkaline hydrolysis was applied for the raspberry and strawberry samples, using an adapted workflow [28]. After incubating the plant material with the BHT solution for $30 \mathrm{~min}$ at $75{ }^{\circ} \mathrm{C}$ under constant shaking, $3.47 \mathrm{~mL}$ IPA and $0.6 \mathrm{~mL}$-hexane were added. Samples were vortexed and put on a shaker for $15 \mathrm{~min}$ at $60{ }^{\circ} \mathrm{C}$. As soon as the samples had reached room temperature $707 \mu \mathrm{L} 1 \mathrm{M} \mathrm{KOH}$ in $\mathrm{MeOH}$ was added and the solution was vortexed. After shaking the samples for $2 \mathrm{~h}$ at $37^{\circ} \mathrm{C}$, they were left at room temperature. Subsequently $100 \%$ formic acid was added until a $\mathrm{pH}$ of $\sim 6-7$ was reached and $1.93 \mathrm{~mL}$ water was added before repeating the incubation step.

\subsubsection{Centrifugation, Drying and Reconstitution}

Irrespective of the extraction strategy, the warm samples were centrifuged at $1000 \mathrm{rpm}$ for $10 \mathrm{~min}$ at $4{ }^{\circ} \mathrm{C}$ and the supernatant was transferred into a separate glass vial. The solvent was evaporated to dryness overnight in a Genevac EZ-2 Series Personal Evaporator (SP Scientific, Ipswich, UK) and the dried residue was reconstituted in 2 mL IPA: $\mathrm{H}_{2} \mathrm{O}$ (65:35) [13]. Samples were vortexed prior and after ultrasonication at $30{ }^{\circ} \mathrm{C}$ for $15 \mathrm{~min}$. Subsequently, $500 \mu \mathrm{L}$ of this solution was filtered directly into HPLC vials through a ClariStep filter (Sartorius, Vienna, Austria). Pools were prepared separately for each plant by pipetting $50 \mu \mathrm{L}$ of each biological replicate into a separate HPLC vial. A quality control pool was prepared by combining $30 \mu \mathrm{L}$ of the pooled samples.

\subsection{Reversed-Phase Chromatography}

Liquid chromatography was performed using a C18 Acquity UHPLC HSS T3 reversed phase column $(2.1 \times 150 \mathrm{~mm}, 100 \AA, 1.8 \mu \mathrm{m}$, Waters, Vienna, Austria) equipped with a VanGuard Pre-column $\left(2.1 \times 5 \mathrm{~mm}, 100 \AA, 1.8 \mu \mathrm{m}\right.$, Waters, Vienna, Austria) at a column temperature of $40^{\circ} \mathrm{C}$. The flow rate was $0.25 \mathrm{~mL} / \mathrm{min}$ and the backpressure was 460 bar at the starting conditions. Gradient elution with a total runtime of 30 min was performed using the solvent $\mathrm{A}$ : $\mathrm{ACN}: \mathrm{H}_{2} \mathrm{O}(3: 2, v / v)$ and the solvent $\mathrm{B}$ : IPA:ACN $(9: 1, v / v)$, both of which contained $0.1 \%$ formic acid and $10 \mathrm{mM}$ ammonium formate.

The gradient can be described as follows: 0-2 $\min 30 \% \mathrm{~B}, 2-3 \mathrm{~min}$ ramp to 55\% B, 3-17 min ramp to $67 \% \mathrm{~B}, 17-22 \mathrm{~min}$ ramp to $100 \% \mathrm{~B}, 22-26 \mathrm{~min} 100 \% \mathrm{~B}$, followed by an equilibration step from 26 to 30 min using 30\% B. A Vanquish Duo UHPLC system (Thermo Fisher Scientific, Germering, Germany) was used and injections were performed with an autosampler. An injection volume of $10 \mu \mathrm{L}$ was chosen and the injector needle was flushed with 75\% IPA and 1\% formic acid in between the injections.

\subsection{High-Resolution Mass Spectrometry}

The LC system was coupled to a Q Exactive HF (Thermo Fisher Scientific, Bremen, Germany) high resolution mass spectrometer, applying a HESI ion source with an S-lens RF level of 45. Measurements were carried out in positive and negative modes using different parameters. The following settings were applied in positive mode: spray voltage: $3.5 \mathrm{kV}$, capillary temperature $220{ }^{\circ} \mathrm{C}$, sheath gas flow rate: 30 , and auxiliary flow rate: 5 . In negative mode parameters were adapted as follows: spray voltage: $2.8 \mathrm{kV}$, capillary temperature $250{ }^{\circ} \mathrm{C}$, sheath gas flow rate: 35 (a.u.), and auxiliary flow rate: 10 (a.u.). The top 10 data-dependent MS2 spectra were obtained at a scan range of 500 to $3000 \mathrm{~m} / \mathrm{z}$ with HCD using normalized collision energies of 35 (+35 in positive ion mode, -35 in negative mode), an MS1 resolution of 15,000 or 30,000 with an AGC target of 1e6 and MS2 resolution of 15,000 with an AGC target of 1e5. MS2 spectra were acquired based on an inclusion list ("do not pick others" option) containing the GIPC series $0-F(m / z$ values were calculated using enviPat Web 2.4 [29]). A more 
comprehensive picture of the GIPC composition of the analyzed plant material was obtained using several rounds of automatically generated exclusions lists for the sample pools [30].

\subsection{Data Analysis}

The GIPC assignment was performed using LDA (version 2.8.0) [17]; corresponding settings (Table A3), mass lists (Tables S3 and S4) and decision rule sets for series A (Folder S1) can be found in the Appendix A and Supplementary Materials. The correct GIPC annotation was ensured by a manual inspection of the results. MS1-based relative quantification of annotated GIPCs was performed with Skyline [25]. Total areas were divided by the corresponding calculated dry weights and areas of the IS, resulting in normalized ratios per $\mathrm{g}$ dry weight, of which the average was taken based on the number of replicates ( 3 for salad and spinach, 4 for strawberries and raspberries). More information can be found in Appendix B.

Supplementary Materials: The following are available online at http://www.mdpi.com/2218-1989/10/9/375/s1, Table S1. Application of the ECN model to ensure correct GIPC annotation, Table S2. List of annotated GIPCs including all detected MS2 fragments, Table S3. LDA mass list used for automated annotation of the series A GIPCs in positive mode, Table S4. LDA mass list used for automated annotation of the series A GIPCs in negative mode, Table S5. List of $[\mathrm{M}+\mathrm{H}]^{+}$precursors comprising the GIPC series $0-\mathrm{F}$, Table S6. List of $[\mathrm{M}-\mathrm{H}]^{-}$precursors comprising the GIPC series 0-F, Folder S1. Fragmentation rules for GIPC analysis by LDA.

Author Contributions: Conceptualization, E.R.; methodology, L.P. and E.R.; software, L.P., S.S., N.T. and J.H.; validation, L.P., E.R. and J.H.; formal analysis, L.P., S.S., N.T. and E.R.; investigation, L.P. and E.R.; resources, E.R. and G.K.; data curation, L.P. and E.R.; writing-original draft preparation, E.R. and L.P.; writing-review and editing, E.R., L.P., J.H. and G.K.; visualization, L.P.; supervision, E.R.; project administration, E.R. and G.K. All authors have read and agreed to the published version of the manuscript.

Funding: This research received no external funding.

Acknowledgments: This work was supported by the University of Vienna, the Faculty of Chemistry, the Vienna Metabolomics Center (VIME; http://metabolomics.univie.ac.at/), the research platform Chemistry Meets Microbiology and the Mass Spectrometry Centre of the University of Vienna. The authors thank (1) the Department of Food Chemistry and Toxicology (University of Vienna) for sharing their sample preparation equipment, (2) Sophia Mundigler for the preparation of a literature database for glycolipid fragmentation (3) Martin Schaier for graphical abstract support, as well as (4) all members of the Koellensperger lab (University of Vienna) for continuous support.

Conflicts of Interest: The authors declare no conflict of interest. 


\section{Appendix A}

Table A1. Overview of GIPCs annotated in salad, spinach, strawberries and raspberries. The precursor ion $m / z$ and retention times are listed as provided by the LDA display results function. In cases where only matching retention times but no $\mathrm{m} / \mathrm{z}$ were explicitly shown by the LDA, because only one MS2 spectrum was annotated (level 3, level $3^{* *}$ ), corresponding values (marked with an asterisk*) were manually assigned at the peak maximum using Thermo Scientific FreeStyle. In the Level column the levels of identification are listed. For all annotated GIPCs, accurate mass and retention times were observed. Their level of identification depends on the ddMS2 spectra. Level 2 annotation is based on the ddMS2 spectra in both ion modes. For level 3, ddMS2 spectra with characteristic fragments could only be detected in one ion mode. Putative hits, which cannot be annotated with such high confidence, because they were only observed with a sugar fragment in positive mode, but did not show the $[\mathrm{IP}]^{+} /[\mathrm{IP}+\mathrm{Na}]^{+}$fragment, are listed as level $3^{* *}$ at the end of the table and were not included in Figure 3 .

\begin{tabular}{|c|c|c|c|c|c|c|c|c|}
\hline Composition & Plant & $\begin{array}{c}m / z \\
{[\mathbf{M}-\mathbf{H}]^{-}}\end{array}$ & $\begin{array}{c}m / z \\
{[\mathbf{M}+\mathbf{H}]^{+}}\end{array}$ & Rt_neg [min] & Rt_pos [min] & Level & Normalized Ratio/g dw $\left[\mathrm{g}^{-1}\right]$ & $\mathrm{CV}[\%]$ \\
\hline A-NAc-t18:1/h16:0 & Spinach & 1189.6246 & 1191.6388 & 8.00 & 8.00 & 2 & 3.15 & 25 \\
\hline A-NAc-t18:1/h16:0 & Salad & 1189.6246 & 1191.6397 & 8.01 & 8.00 & 2 & 2.10 & 28 \\
\hline A-NAc-t18:1/h22:0 & Spinach & 1273.7179 & 1275.7359 & 14.25 & 14.25 & 2 & 27.43 & 19 \\
\hline A-NAc-t18:1/h22:0 & Salad & 1273.7193 & 1275.7344 & 14.19 & 14.26 & 2 & 3.57 & 44 \\
\hline A-NAc-t18:1/h22:0 & Raspberry & 1273.7172 & 1275.7341 & 14.21 & 14.19 & 2 & 0.94 & 6 \\
\hline A-NAc-t18:1/h23:0 & Spinach & 1287.7325 & 1289.7504 & 15.64 & 15.65 & 2 & 11.83 & 21 \\
\hline A-NAc-t18:0/h24:0 & Raspberry & 1303.7648 & 1305.7813 & 17.94 & 18.17 & 2 & 2.13 & 4 \\
\hline A-NAc-t18:1/h24:0 & Spinach & 1301.7502 & 1303.7657 & 16.98 & 16.98 & 2 & 70.89 & 21 \\
\hline A-NAc-t18:1/h24:0 & Salad & 1301.7498 & 1303.7637 & 16.98 & 16.94 & 2 & 17.23 & 32 \\
\hline A-NAc-t18:1/h24:0 & Raspberry & 1301.7491 & 1303.7649 & 16.96 & 16.94 & 2 & 3.40 & 5 \\
\hline A-NAc-t18:2/h24:0 & Raspberry & 1299.7322 & 1301.749 & 14.98 & 14.97 & 2 & 1.27 & 3 \\
\hline A-NAc-t18:1/h25:0 & Spinach & 1315.7652 & 1317.7811 & 18.41 & 18.41 & 2 & 11.08 & 20 \\
\hline A-NAc-t18:1/h26:0 & Spinach & 1329.7811 & 1331.797 & 19.75 & 19.75 & 2 & 20.54 & 16 \\
\hline A-NH2-t34:0 & Raspberry & 1133.6346 & 1135.6506 & 10.91 & 10.92 & 2 & 1.09 & 6 \\
\hline A-NH2-d34:1 & Raspberry & 1115.6248 * & 1117.6415 & 10.89 & 10.90 & 3 & 1.85 & 7 \\
\hline A-NH2-t34:1 & Raspberry & $1131.6196^{*}$ & 1133.6365 & 10.27 & 10.27 & 3 & 0.75 & 8 \\
\hline A-NH2-t18:1/h16:0 & Salad & 1147.6148 & 1149.6302 & 8.64 & 8.52 & 2 & 6.46 & 5 \\
\hline A-NH2-q40:0 & Salad & 1233.7225 * & 1235.7372 & 16.82 & 16.76 & 3 & 3.87 & 27 \\
\hline A-NH2-t18:0/h22:0 & Raspberry & 1233.7242 & 1235.7389 & 16.64 & 16.55 & 2 & 3.33 & 7 \\
\hline A-NH2-t18:1/h22:0 & Spinach & $1231.7058^{*}$ & 1233.7229 & 15.56 & 15.52 & 3 & 1.87 & 18 \\
\hline A-NH2-t18:1/h22:0 & Salad & 1231.7083 & 1233.7226 & 15.51 & 15.49 & 2 & 10.45 & 11 \\
\hline A-NH2-t18:1/h22:0 & Raspberry & 1231.7082 & 1233.7234 & 15.41 & 15.25 & 2 & 3.87 & 7 \\
\hline A-NH2-t18:2/h22:0 & Raspberry & $1229.6945 *$ & 1231.7076 & 13.42 & 13.42 & 3 & 1.01 & 4 \\
\hline A-NH2-t18:1/h23:0 & Salad & $1245.7242 *$ & 1247.7400 & 16.78 & 16.87 & 3 & 4.04 & 15 \\
\hline A-NH2-t18:1/h23:0 & Raspberry & 1245.7248 & 1247.7388 & 16.89 & 16.85 & 2 & 2.79 & 7 \\
\hline A-NH2-t18:0/h24:0 & Raspberry & 1261.7551 & 1263.7704 & 19.62 & 19.64 & 2 & 6.92 & 9 \\
\hline A-NH2-t18:0/h24:0 & Salad & $1261.7531^{*}$ & 1263.7701 & 19.82 & 19.53 & 3 & 3.80 & 27 \\
\hline
\end{tabular}


Table A1. Cont.

\begin{tabular}{|c|c|c|c|c|c|c|c|c|}
\hline Composition & Plant & $\begin{array}{c}m / z \\
{[\mathbf{M}-\mathbf{H}]^{-}}\end{array}$ & $\begin{array}{c}m / z \\
{[\mathbf{M}+\mathbf{H}]^{+}}\end{array}$ & Rt_neg [min] & Rt_pos [min] & Level & Normalized Ratio/g dw $\left[\mathrm{g}^{-1}\right]$ & $\mathrm{CV}[\%]$ \\
\hline A-NH2-t18:1/h24:0 & Spinach & 1259.7391 & 1261.7565 & 18.32 & 18.30 & 2 & 5.06 & 18 \\
\hline A-NH2-t18:1/h24:0 & Salad & 1259.7400 & 1261.7554 & 18.52 & 18.44 & 2 & 32.00 & 6 \\
\hline A-NH2-t18:1/h24:0 & Raspberry & 1259.7400 & 1261.7543 & 18.46 & 18.42 & 2 & 12.28 & 8 \\
\hline A-NH2-t18:2/h24:0 & Raspberry & 1257.7231 & 1259.7397 & 15.98 & 16.25 & 2 & 4.22 & 8 \\
\hline A-NH2-t18:1/h25:0 & Raspberry & $1273.7539 *$ & 1275.7692 & 19.55 & 19.78 & 3 & 1.55 & 11 \\
\hline A-NH2-q43:2 & Raspberry & 1271.7419 * & 1273.7571 & 17.71 & 17.73 & 3 & 0.87 & 9 \\
\hline A-OH-t34:0 & Raspberry & 1134.6192 & 1136.6369 & 10.21 & 10.21 & 2 & 0.58 & 8 \\
\hline A-OH-t18:0/h16:0 & Salad & 1150.6148 & 1152.6313 & 8.84 & 8.83 & 2 & 1.93 & 0 \\
\hline A-OH-t18:1/h16:0 & Salad & 1148.5984 & 1150.6138 & 8.14 & 8.13 & 2 & 10.27 & 16 \\
\hline A-OH-q34:1 & Strawberry & 1148.5982 & 1150.6144 & 8.11 & 8.13 & 2 & 1.51 & 6 \\
\hline A-OH-q40:0 & Salad & 1234.7095 & 1236.7239 * & 15.66 & 15.60 & 3 & 3.00 & 2 \\
\hline $\mathrm{A}-\mathrm{OH}-\mathrm{t} 18: 0 / \mathrm{h} 22: 0$ & Raspberry & 1234.7084 & 1236.7247 & 15.36 & 15.63 & 2 & 1.37 & 6 \\
\hline A-OH-t18:1/h22:0 & Salad & 1232.6921 & 1234.7071 & 14.47 & 14.44 & 2 & 15.16 & 19 \\
\hline A-OH-t18:1/h22:0 & Strawberry & 1232.6921 & 1234.7083 & 14.25 & 14.43 & 2 & 3.69 & 2 \\
\hline A-OH-t18:1/h22:0 & Raspberry & 1232.6924 & 1234.7055 & 14.43 & 14.43 & 2 & 1.44 & 6 \\
\hline A-OH-q41:0 & Strawberry & 1248.7232 & 1250.7395 * & 17.01 & 16.98 & 3 & 0.83 & 2 \\
\hline A-OH-t18:1/h23:0 & Raspberry & 1246.7076 & 1248.78221 & 15.82 & 15.77 & 2 & 1.46 & 7 \\
\hline A-OH-t18:1/h23:0 & Salad & 1246.7079 & 1248.7209 & 15.85 & 15.84 & 2 & 4.45 & 18 \\
\hline A-OH-t18:1/h23:0 & Strawberry & 1246.7076 & 1248.7238 & 15.53 & 15.76 & 2 & 8.20 & 1 \\
\hline A-OH-t18:0/h24:0 & Salad & 1262.7397 & 1264.7564 & 18.55 & 18.50 & 2 & 12.30 & 4 \\
\hline A-OH-t18:0/h24:0 & Raspberry & 1262.7385 & 1264.754 & 18.51 & 18.53 & 2 & 2.58 & 6 \\
\hline A-OH-t18:0/h24:0 & Strawberry & 1262.7387 & 1264.7553 & 18.44 & 18.32 & 2 & 4.53 & 1 \\
\hline A-OH-t18:1/h24:0 & Salad & 1260.7237 & 1262.7389 & 17.30 & 17.26 & 2 & 50.77 & 14 \\
\hline A-OH-t18:1/h24:0 & Strawberry & 1260.7227 & 1262.7391 & 17.26 & 17.00 & 2 & 22.15 & 2 \\
\hline A-OH-t18:1/h24:0 & Raspberry & 1260.7240 & 1262.7388 & 17.25 & 17.23 & 2 & 3.89 & 6 \\
\hline A-OH-q42:2 & Strawberry & 1258.7073 & 1260.7238 * & 15.21 & 15.22 & 3 & 1.22 & 2 \\
\hline A-OH-t18:1/h24:1 & Salad & 1258.7067 & 1260.7243 & 14.36 & 14.33 & 2 & 2.04 & 10 \\
\hline $\mathrm{A}-\mathrm{OH}-\mathrm{t} 18: 2 / \mathrm{h} 24: 0$ & Raspberry & 1258.7082 & 1260.7234 & 15.23 & 15.23 & 2 & 1.47 & 5 \\
\hline A-OH-t18:0/h25:0 & Strawberry & 1276.7542 & 1278.7687 & 19.80 & 19.80 & 2 & 1.33 & 5 \\
\hline A-OH-t18:1/h25:0 & Salad & 1274.7387 & 1276.7533 & 18.76 & 18.75 & 2 & 3.72 & 11 \\
\hline A-OH-t18:1/h25:0 & Strawberry & 1274.7371 & 1276.7557 & 18.42 & 18.48 & 2 & 5.83 & 2 \\
\hline A-OH-t18:1/h25:0 & Raspberry & 1274.7381 & 1276.7548 & 18.71 & 18.73 & 2 & 0.84 & 9 \\
\hline A-OH-t18:1/h26:0 & Salad & 1288.7553 & 1290.7706 & 20.01 & 20.01 & 2 & 8.65 & 18 \\
\hline A-OH-t18:1/h26:0 & Strawberry & 1288.7540 * & 1290.7697 & 19.76 & 19.94 & 3 & 5.49 & 2 \\
\hline A-NH2-d18:2/16:0 & Raspberry & $1113.6100 *$ & 1115.6255 & 9.89 & 9.89 & $3^{* *}$ & 0.57 & 10 \\
\hline A-NH2-q41:0 & Raspberry & 1247.7383 * & 1249.7544 & 18.16 & 18.17 & $3^{* *}$ & 2.03 & 7 \\
\hline A-NH2-q41:2 & Raspberry & 1243.7076 * & 1245.7232 & 14.77 & 14.80 & $3^{* *}$ & 0.87 & 7 \\
\hline
\end{tabular}




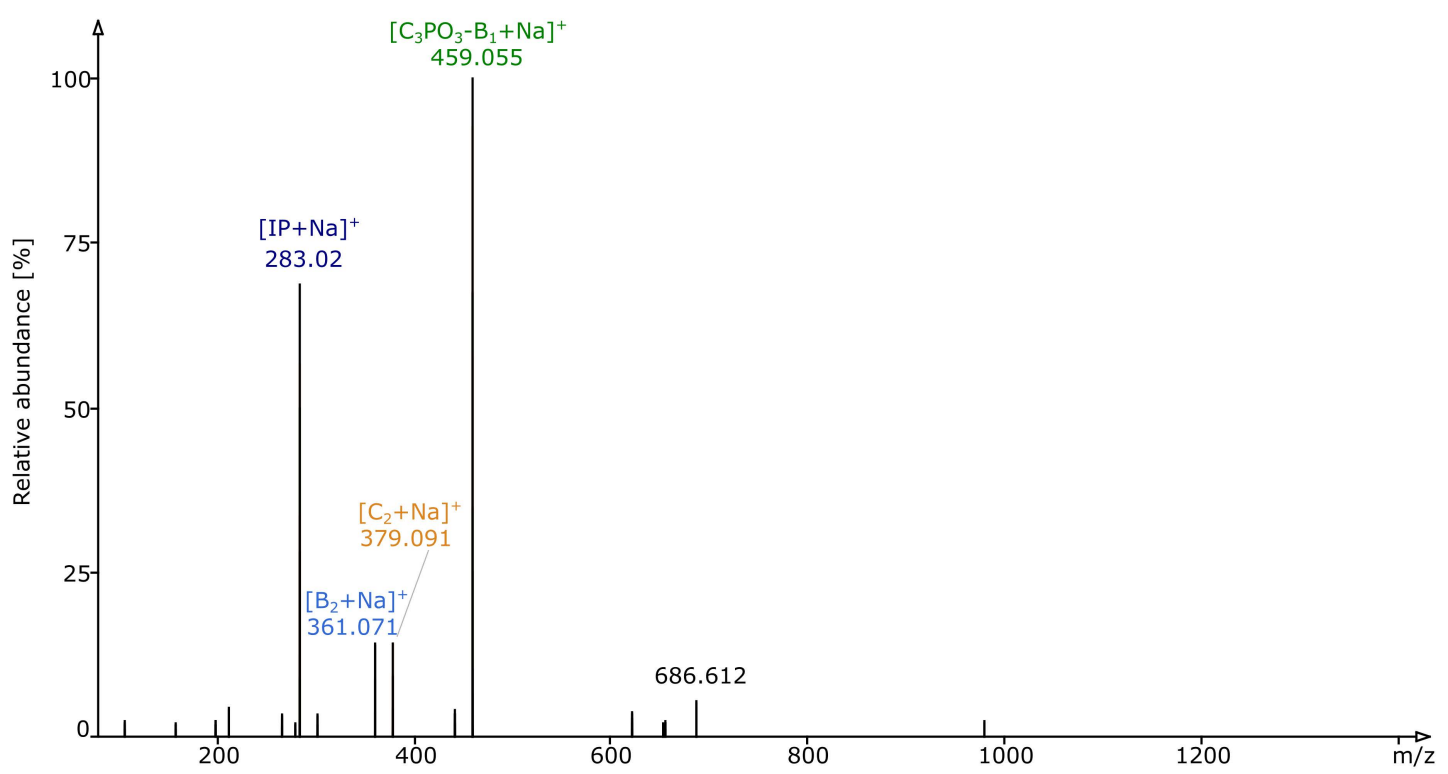

Figure A1. The ddMS2 spectrum of the $[\mathrm{M}+\mathrm{Na}]^{+}$of GIPC A-OH-t18:1/h24:0 (m/z 1284.7193, Rt $17.30 \mathrm{~min})$, measured in positive ion mode, showing the characteristic [IP $+\mathrm{Na}]^{+}$and additional sugar fragments.

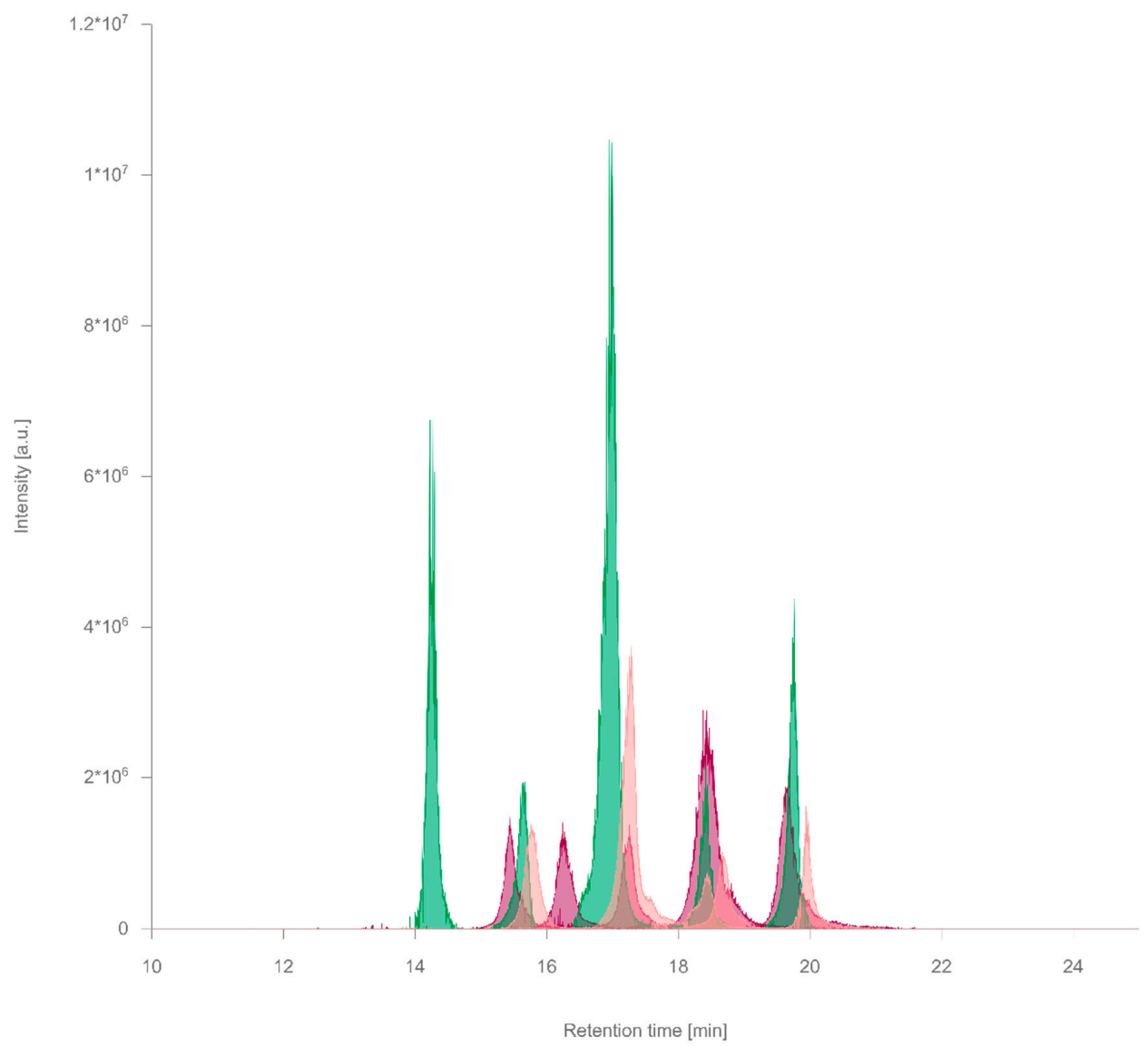

Figure A2. Comparison of the RP-HRMS/MS GIPC profiles in spinach (green), strawberry (rose) and raspberry (dark-red), showing the five most abundant GIPCs found in each plant sample measured in positive ion mode (detailed information can be found in Table A1). 

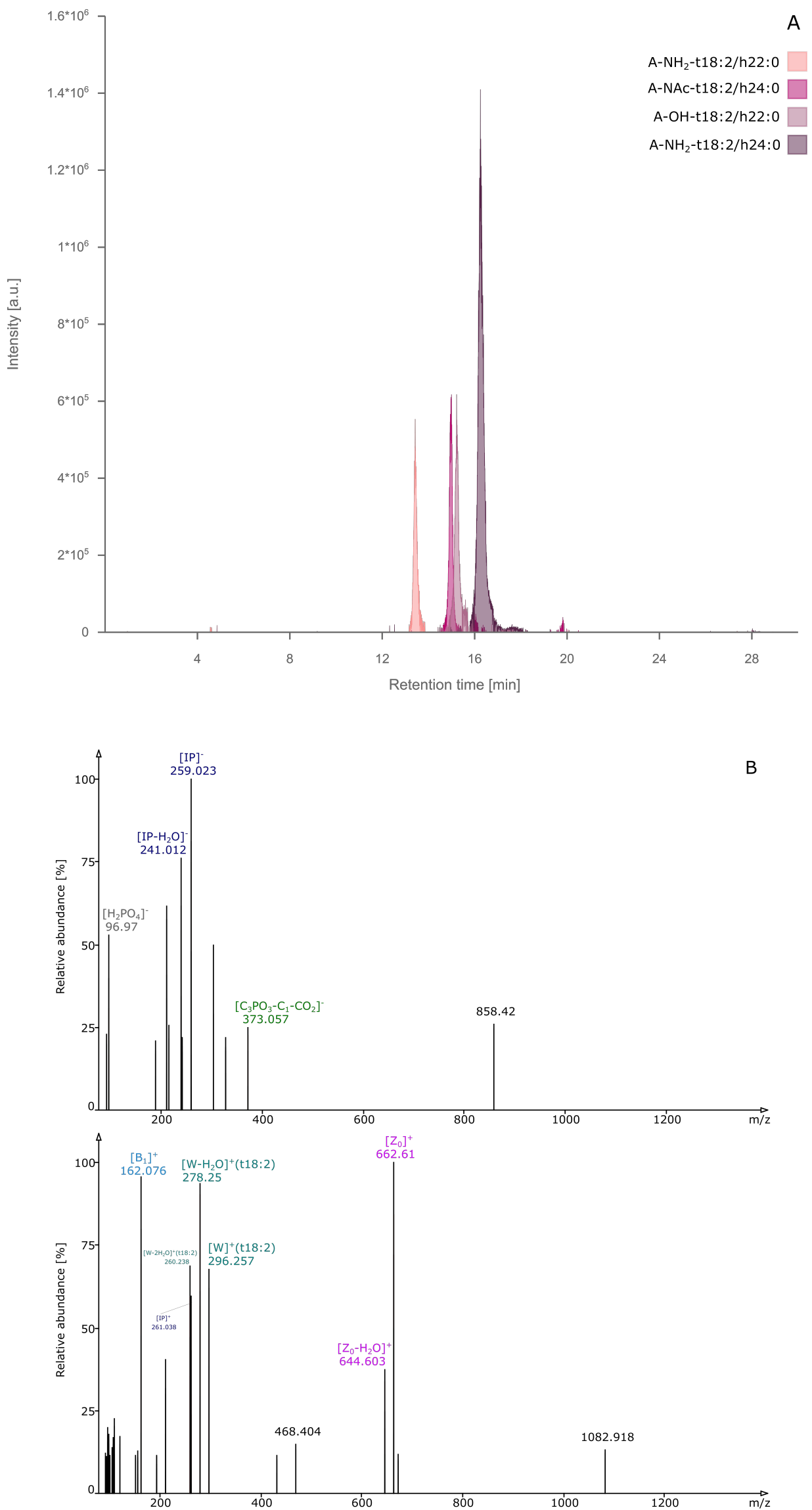

Figure A3. Cont. 

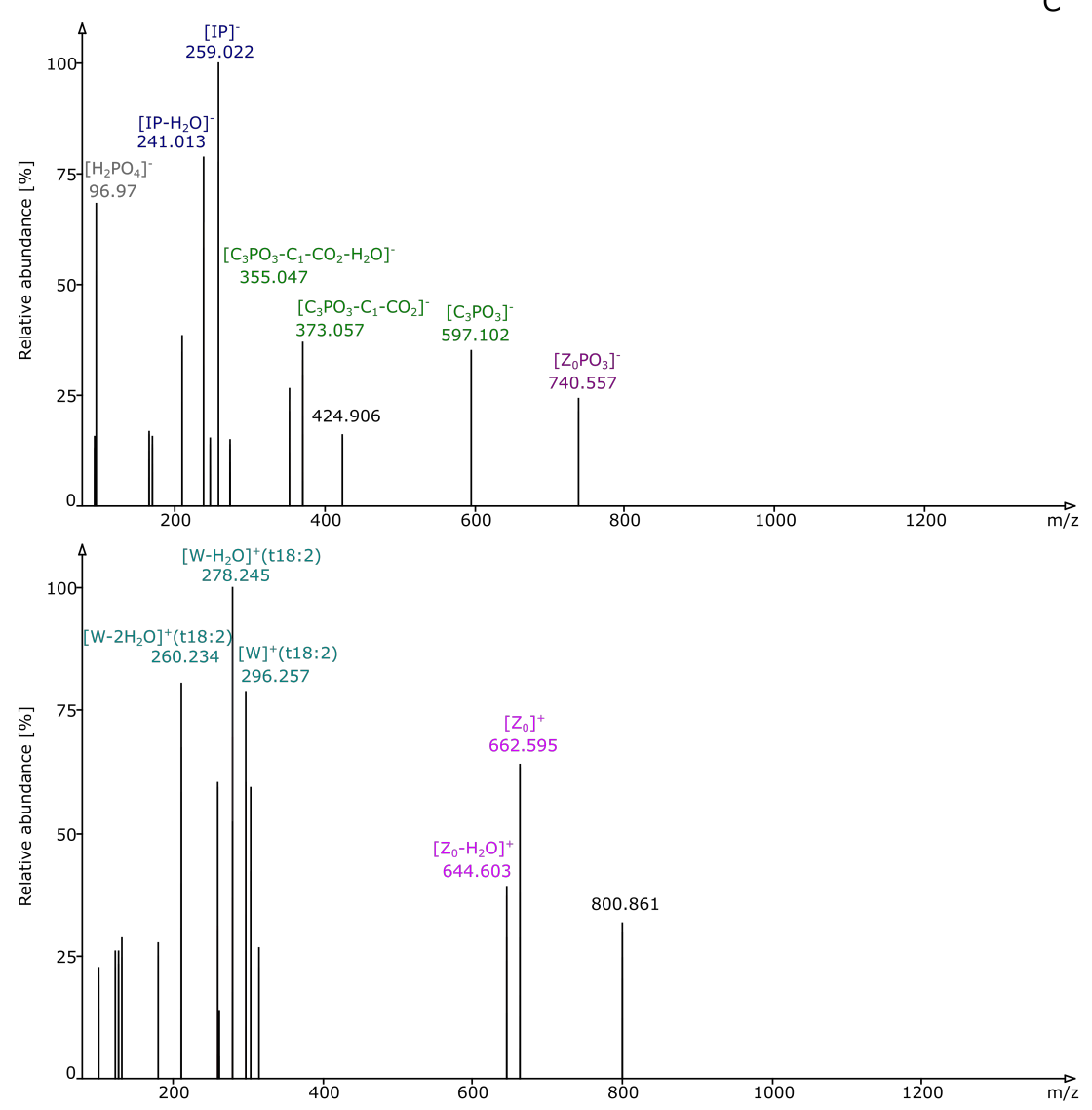

Figure A3. (A) Extracted ion chromatograms of GIPCs in positive ion mode, having a t18:2 LCB annotation on levels 2 and 3 in raspberries (A-NAc-t18:2/h24:0, A- $\mathrm{NH}_{2}-\mathrm{t} 18: 2 \mathrm{~h} 22: 0$ and h24:0, as well as A-OH-t18:2/h24:0). The ddMS2 spectra of GIPCs A-NH $-\mathrm{t} 18: 2 / \mathrm{h} 24: 0$ at the retenion time of $16.1 \mathrm{~min}$ (B) A-OH-t18:2/h24:0 at the retention time of $15.2 \mathrm{~min}$ and (C) in negative and positive mode, showing characteristic fragments.

Table A2. The description of the plant samples, including plant species, origin, number of biological replicates, and the average fresh- and dry weights [g]. The extraction of GIPCs from strawberries and raspberries was performed one day after the collection was performed (28 June 2020).

\begin{tabular}{ccccc}
\hline Plant Species & Origin & Replicates & Fresh Weight [g] & Dry Weight [g] \\
\hline Salad & Local supermarket & 4 & $\sim 1$ & $\sim 0.04$ \\
Spinach & Local supermarket & 4 & $\sim 1.2$ & $\sim 0.08$ \\
Strawberries & $47^{\circ} 58^{\prime} \mathrm{N}, 16^{\circ} 6^{\prime}$ O & 5 & $\sim 1.1$ & $\sim 0.10$ \\
Raspberries & $47^{\circ} 58^{\prime} \mathrm{N}, 16^{\circ} 6^{\prime} \mathrm{O}$ & 5 & $\sim 1.1$ & $\sim 0.16$ \\
\hline
\end{tabular}


Table A3. The exemplary LDA parameters and settings used for automated GIPC annotation in negative ion mode.

\begin{tabular}{|c|c|}
\hline Parameter & Setting \\
\hline Time before tol. & $1 \mathrm{~min}$ \\
\hline Time after tol. & $1 \mathrm{~min}$ \\
\hline Rel. Base-peak cutoff & $0.1 \%$ \\
\hline Rt-shift & $0.0 \mathrm{~min}$ \\
\hline $\begin{array}{c}\text { Isotopic quantitation of _ isotopes where _ isotopic peak(s) } \\
\text { have to match }\end{array}$ & 2,1 \\
\hline Find molecules where retention time is unknown & yes \\
\hline LDA-version & 2.8 .0 \\
\hline machineName & OrbiTrap_exactive \\
\hline neutronMass & 1.005 \\
\hline coarseChromMzTolerance & 0.015 \\
\hline MS2 & true \\
\hline basePeakCutoff & 0.1 \\
\hline massShift & 0.0 \\
\hline threeDViewerDefaultTimeResolution & 2 \\
\hline threeDViewerDefaultMZResolution & 0.005 \\
\hline ms2PrecursorTolerance & 0.013 \\
\hline ms2MzTolerance & 0.02 \\
\hline ms2MinIntsForNoiseRemoval & 100 \\
\hline ms2IsobarSCExclusionRatio & 0.01 \\
\hline ms2IsobarSCFarExclusionRatio & 0.1 \\
\hline ms2IsobaricOtherRtDifference & 2.0 \\
\hline chainCutoffValue & 0.01 \\
\hline ms2ChromMultiplicationFactorForInt & 10 \\
\hline threeDViewerMs2DefaultTimeResolution & 1 \\
\hline threeDViewerMs2DefaultMZResolution & 1 \\
\hline maxFileSizeForChromTranslationAtOnce & 500 \\
\hline chromMultiplicationFactorForInt & 1000 \\
\hline chromLowestResolution & 1 \\
\hline chromSmoothRange & 8.0 \\
\hline chromSmoothRepeats & 4 \\
\hline use $3 \mathrm{D}$ & true \\
\hline isotopeCorrection & false \\
\hline removeFromOtherIsotopes & true \\
\hline respectIsotopicDistribution & true \\
\hline checkChainLabelCombinationFromSpeciesName & false \\
\hline useNoiseCutoff & true \\
\hline noiseCutoffDeviationValue & 2.0 \\
\hline scanStep & 2 \\
\hline profileMzRangeExtraction & 0.05 \\
\hline profileTimeTolerance & 5.0 \\
\hline profileIntThreshold & 5.0 \\
\hline broaderProfileTimeTolerance & 3.0 \\
\hline profileSmoothRange & 0.0025 \\
\hline profileSmoothRepeats & 1 \\
\hline profileMeanSmoothRepeats & 2 \\
\hline profileMzMinRange & 0.002 \\
\hline profileSteepnessChange1 & 1.5 \\
\hline profileSteepnessChange2 & 1.8 \\
\hline profileIntensityCutoff1 & 0.15 \\
\hline profileIntensityCutoff2 & 0.2 \\
\hline profileGeneralIntCutoff & 0.03 \\
\hline profilePeakAcceptanceRange & 0.012 \\
\hline profileSmoothingCorrection & 0.0 \\
\hline profileMaxRange & 0.03 \\
\hline smallChromMzRange & 0.004 \\
\hline
\end{tabular}


Table A3. Cont.

\begin{tabular}{|c|c|}
\hline Parameter & Setting \\
\hline smallChromSmoothRepeats & 3 \\
\hline smallChromMeanSmoothRepeats & 0 \\
\hline smallChromSmoothRange & 2.0 \\
\hline smallChromIntensityCutoff & 0.03 \\
\hline broadChromSmoothRepeats & 5 \\
\hline broadChromMeanSmoothRepeats & 0 \\
\hline broadChromSmoothRange & 2 \\
\hline broadChromIntensityCutoff & 0.0 \\
\hline broadChromSteepnessChangeNoSmall & 1.33 \\
\hline broadChromIntensityCutoffNoSmall & 0.05 \\
\hline finalProbeTimeCompTolerance & 0.1 \\
\hline finalProbeMzCompTolerance & $5.0 \mathrm{E}-4$ \\
\hline overlapDistanceDeviationFactor & 1.5 \\
\hline overlapPossibleIntensityThreshold & 0.15 \\
\hline overlapSureIntensityThreshold & 0.7 \\
\hline overlapPeakDistanceDivisor & 3.0 \\
\hline overlapFullDistanceDivisor & 6.0 \\
\hline peakDiscardingAreaFactor & 1000 \\
\hline isotopeInBetweenTime & 30 \\
\hline isoInBetweenAreaFactor & 3.0 \\
\hline isoNearNormalProbeTime & 30 \\
\hline relativeAreaCutoff & 0.05 \\
\hline relativeFarAreaCutoff & 0.05 \\
\hline relativeFarAreaTimeSpace & 30 \\
\hline relativeIsoInBetweenCutoff & 0.5 \\
\hline isoInBetweenMaxTimeDistance & 300 \\
\hline twinPeakMzTolerance & 0.01 \\
\hline closePeakTimeTolerance & 10 \\
\hline twinInBetweenCutoff & 0.95 \\
\hline unionInBetweenCutoff & 0.8 \\
\hline sparseData & false \\
\hline
\end{tabular}

\section{Appendix B}

Automated GIPC annotation was performed using LDA (version 2.8.0) [17] with the settings provided in Table A3. The mass-to-charge ratios included in the mass lists (see Tables S3 and S4) were calculated separately for negative and positive ion modes, with enviPat Web 2.4 [29] and decision rules (see Folder S1) were created based on fragments reported in the literature [12,13]. Please note that the raw data acquired in negative ion mode has to be analyzed using the mass list of Table $\mathrm{S} 4$ and the fragmentation rules ending with '-H.frag', while for positive mode the mass list of Table S3 and corresponding fragmentation rules ('H.frag' and 'Na.frag') should be used. Further information on working with the LDA can be found in [31].

\section{References}

1. Gronnier, J.; Germain, V.; Gouguet, P.; Cacas, J.-L.; Mongrand, S. GIPC: Glycosyl Inositol Phospho Ceramides, the major sphingolipids on earth. Plant Signal. Behav. 2016, 11, e1152438. [CrossRef] [PubMed]

2. Carter, H.E.; Gigg, R.H.; Laws, J.H. Structure of Phytoglyolipide. J. Biol. Chem. 1958, 233, 1309-1314. [PubMed]

3. Cacas, J.; Buré, C.; Grosjean, K.; Gerbeau-Pissot, P.; Lherminier, J.; Rombouts, Y.; Maes, E.; Bossard, C.; Gronnier, J.; Furt, F.; et al. Revisiting Plant Plasma Membrane Lipids in Tobacco: A Focus on Sphingolipids. Plant Physiol. 2016, 170, 367-384. [CrossRef] [PubMed]

4. Carter, H.E.; Strobach, D.R.; Hawthorne, J.N. Biochemistry of the Sphingolipids. XVIII. Complete Structure of Tetrasaccharide Phytoglycolipid. Biochemistry 1969, 8, 383-388. [CrossRef] [PubMed] 
5. Buré, C.; Cacas, J.; Mongrand, S.; Schmitter, J.-M. Characterization of glycosyl inositol phosphoryl ceramides from plants and fungi by mass spectrometry. Anal. Bioanal. Chem. 2014, 406, 995-1010. [CrossRef]

6. LIPID MAPS ${ }^{\circledR}$ Lipidomics Gateway. Available online: https://lipidmaps.org/resources/lipidweb/index.php? page=lipids/sphingo/glyP_ino/index.htm (accessed on 4 August 2020).

7. Buré, C.; Cacas, J.-L.; Wang, F.; Gaudin, K.; Domergue, F.; Mongrand, S.; Schmitter, J.-M. Fast screening of highly glycosylated plant sphingolipids by tandem mass spectrometry. Rapid Commun. Mass Spectrom. 2011, 25, 3131-3145. [CrossRef]

8. Hastings, J.; Owen, G.; Dekker, A.; Ennis, M.; Kale, N.; Muthukrishnan, V.; Turner, S.; Swainston, N.; Mendes, P.; Steinbeck, C. ChEBI in 2016: Improved services and an expanding collection of metabolites. Nucleic Acids Res. 2016, 44, D1214-D1219. [CrossRef]

9. Sud, M.; Fahy, E.; Cotter, D.; Brown, A.; Dennis, E.A.; Glass, C.K.; Merrill, A.H.; Murphy, R.C.; Raetz, C.R.H.; Russell, D.W.; et al. LMSD: LIPID MAPS structure database. Nucleic Acids Res. 2007, 35, 527-532. [CrossRef]

10. Barrientos, R.C.; Zhang, Q. Recent advances in the mass spectrometric analysis of glycosphingolipidome-A review. Anal. Chim. Acta 2020. [CrossRef]

11. Markham, J.E.; Jaworski, J.G. Rapid measurement of sphingolipids from Arabidopsis thaliana by reversed-phase high-performance liquid chromatography coupled to electrospray ionization tandem mass spectrometry. Rapid Commun. Mass Spectrom. 2007, 21, 1304-1314. [CrossRef]

12. Blaas, N.; Humpf, H.U. Structural profiling and quantitation of glycosyl inositol phosphoceramides in plants with fourier transform mass spectrometry. J. Agric. Food Chem. 2013, 61, 4257-4269. [CrossRef] [PubMed]

13. Cacas, J.; Buré, C.; Furt, F.; Maalouf, J.; Badoc, A.; Cluzet, S.; Schmitter, J.; Antajan, E.; Mongrand, S. Biochemical survey of the polar head of plant glycosylinositolphosphoceramides unravels broad diversity. Phytochemistry 2013, 96, 191-200. [CrossRef] [PubMed]

14. Domon, B.; Costello, C.E. Structure Elucidation of Glycosphingolipids and Gangliosides Using High-Performance Tandem Mass Spectrometry. Biochemistry 1988, 27, 1534-1543. [CrossRef] [PubMed]

15. Domon, B.; Costello, C.E. A systematic nomenclature for carbohydrate fragmentations in FAB-MS/MS spectra of glycoconjugates. Glycoconj. J. 1988, 5, 397-409. [CrossRef]

16. Hsu, F.; Turk, J. Glycosphingolipids as Lithiated Adducts by Dissociation on a Triple Stage Quadrupole Instrument. J. Am. Soc. Mass Spectrom. 2001, 12, 61-79. [CrossRef]

17. Hartler, J.; Triebl, A.; Ziegl, A.; Trötzmüller, M.; Rechberger, G.N.; Zeleznik, O.A.; Zierler, K.A.; Torta, F.; Cazenave-Gassiot, A.; Wenk, M.R.; et al. Deciphering lipid structures based on platform-independent decision rules. Nat. Methods 2017, 14, 1171-1174. [CrossRef]

18. Markham, J.E.; Li, J.; Cahoon, E.B.; Jaworski, J.G. Separation and Identification of Major Plant Sphingolipid Classes from Leaves. J. Biol. Chem. 2006, 281, 22684-22694. [CrossRef]

19. Dugo, P.; Cacciola, F.; Kumm, T.; Dugo, G.; Mondello, L. Comprehensive multidimensional liquid chromatography: Theory and applications. J. Chromatogr. A 2008, 1184, 353-368. [CrossRef]

20. Lísa, M.; Holčapek, M. Triacylglycerols profiling in plant oils important in food industry, dietetics and cosmetics using high-performance liquid chromatography-atmospheric pressure chemical ionization mass spectrometry. J. Chromatogr. A 2008, 1198-1199, 115-130. [CrossRef]

21. Ovčačíková, M.; Lísa, M.; Cífková, E.; Holčapek, M. Retention behavior of lipids in reversed-phase ultrahigh-performance liquid chromatography-electrospray ionization mass spectrometry. J. Chromatogr. A 2016, 1450, 76-85. [CrossRef]

22. Hartler, J.; Trötzmüller, M.; Chitraju, C.; Spener, F.; Köfeler, H.C.; Thallinger, G.G. Lipid Data Analyzer: Unattended identification and quantitation of lipids in LC-MS data. Bioinformatics 2011, 27, 572-577. [CrossRef] [PubMed]

23. LIPID MAPS®Lipidomics Gateway. Available online: https://www.lipidmaps.org/data/LMSDRecord.php? LMID=LMSP03030005 (accessed on 15 March 2020).

24. Salek, R.M.; Steinbeck, C.; Viant, M.R.; Goodacre, R.; Dunn, W.B. The role of reporting standards for metabolite annotation and identification in metabolomic studies. Gigascience 2013, 2, 2047-217X-2-13. [CrossRef] [PubMed]

25. Adams, K.J.; Pratt, B.; Bose, N.; Dubois, L.G.; St. John-Williams, L.; Perrott, K.M.; Ky, K.; Kapahi, P.; Sharma, V.; Maccoss, M.J.; et al. Skyline for Small Molecules: A Unifying Software Package for Quantitative Metabolomics. J. Proteome Res. 2020, 19, 1447-1458. [CrossRef] [PubMed]

26. Metabolomics Society. Available online: http://metabolomicssociety.org/ (accessed on 14 July 2020). 
27. Shiva, S.; Enninful, R.; Roth, M.R.; Tamura, P.; Jagadish, K.; Welti, R. An efficient modified method for plant leaf lipid extraction results in improved recovery of phosphatidic acid. Plant Methods 2018, 14, 1-8. [CrossRef]

28. Peng, B.; Weintraub, S.T.; Coman, C.; Ponnaiyan, S.; Sharma, R.; Tews, B.; Winter, D.; Ahrends, R. A Comprehensive High-Resolution Targeted Workflow for the Deep Profiling of Sphingolipids. Anal. Chem. 2017, 89, 12480-12487. [CrossRef] [PubMed]

29. Loos, M.; Gerber, C.; Corona, F.; Hollender, J.; Singer, H. Accelerated Isotope Fine Structure Calculation Using Pruned Transition Trees. Anal. Chem. 2015, 87, 5738-5744. [CrossRef] [PubMed]

30. Koelmel, J.P.; Kroeger, N.M.; Gill, E.L.; Ulmer, C.Z.; Bowden, J.A.; Patterson, R.E.; Yost, R.A.; Garrett, T.J. Expanding Lipidome Coverage Using LC-MS/MS Data-Dependent Acquisition with Automated Exclusion List Generation. J. Am. Soc. Mass Spectrom. 2017, 28, 908-917. [CrossRef]

31. LDA User Manual. Available online: http://genome.tugraz.at/lda2/2.6/LDA_2.6.pdf (accessed on 17 September 2020).

(C) 2020 by the authors. Licensee MDPI, Basel, Switzerland. This article is an open access article distributed under the terms and conditions of the Creative Commons Attribution (CC BY) license (http://creativecommons.org/licenses/by/4.0/). 